\title{
Neutron inelastic scattering measurements on the stable isotopes of titanium
}

\author{
A. Olacel, ${ }^{1,{ }^{*}}$ F. Belloni, ${ }^{2}$ C. Borcea, ${ }^{1}$ M. Boromiza,${ }^{1,3}$ P. Dessagne, ${ }^{4}$ G. Henning,${ }^{4}$ M. Kerveno, ${ }^{4}$ A. Negret, ${ }^{1}$ M. Nyman, ${ }^{2}$ \\ E. Pirovano, ${ }^{2}$ and A. J. M. Plompen ${ }^{2}$ \\ ${ }^{1}$ Horia Hulubei National Institute for Physics and Nuclear Engineering, Reactorului 30, 077125 Bucharest-Măgurele, Romania \\ ${ }^{2}$ European Commission, Joint Research Centre, Retieseweg 111, B-2440 Geel, Belgium \\ ${ }^{3}$ University of Bucharest, Faculty of Physics, Atomistilor 405, 077125, Bucharest-Măgurele, Romania \\ ${ }^{4}$ Université de Strasbourg, CNRS, IPHC UMR 7178, F-67000 Strasbourg, France
}

(Received 24 May 2017; published 31 July 2017)

\begin{abstract}
The results of a neutron inelastic scattering experiment performed at the Geel Electron Linear Accelerator pulsed white neutron source of the European Commission Joint Research Centre are reported. The neutrons with energies up to $18 \mathrm{MeV}$ interacted with a ${ }^{\text {nat }} \mathrm{Ti}$ sample and the $\gamma$ rays resulting from inelastic scattering reactions on the stable isotopes were detected using the Gamma Array for Inelastic Neutron Scattering (GAINS) spectrometer. We were able to measure the $\gamma$-production cross sections for 21 transitions in the five stable Ti isotopes. From these, the level cross sections and the total inelastic cross sections were determined. Our experimental results are compared with theoretical calculations performed using the TALYS 1.8 code, evaluated nuclear data libraries, and also with previously reported results.
\end{abstract}

DOI: 10.1103/PhysRevC.96.014621

\section{INTRODUCTION}

An obvious correlation exists between the increase of energy consumption and the evolution of the human society [1]. This increase has to be compensated by a higher production, and one of the most important solutions is the nuclear option, in spite of some disadvantages (e.g., limited availability of nuclear fuel, radioactive waste, Research and Development costs, the danger of proliferation). A new generation of nuclear reactors (Gen IV) [2] addresses these disadvantages. For the development of these facilities accurate knowledge of the nuclear reactions taking place inside the reactors is required.

The precise knowledge of the neutron-induced reactions cross sections, in particular inelastic scattering and $(n, 2 n)$ reactions, is required because they have a direct impact on the value of the criticality coefficient of fission reactors [3]. Inelastic scattering plays a significant role in slowing down the neutrons while the knowledge of the $(n, 2 n)$ reactions is important because of the neutron multiplication and the creation of new isotopes in the reactor core. Many of the current demands for inelastic scattering and $(n, x n)$ measurements require a total uncertainty lower than 5\% [4].

An important motivation to perform high precision neutron inelastic scattering measurements on titanium arises from the the fact that $983.5 \mathrm{keV} \gamma$ ray in ${ }^{48} \mathrm{Ti}$ is one of the leading candidates to establish a recognized $\gamma$-ray reference cross section for neutron-induced reactions [5]. The existence of a reference cross section for neutron-induced reactions is essential given the difficulties of performing a precise neutron flux determination. Also, a $\gamma$-ray production standard for inelastic neutron scattering will facilitate reliable measurements of inelastic scattering cross sections by $\gamma$ detection provided it meets a number of criteria which are shortly described in

*aolacel@tandem.nipne.ro the end of this section. The uncertainty of the standard cross section determines the lower limit of the experimental cross section uncertainties in such applications. There are only a few experimental values of the neutron inelastic cross sections on the stable isotopes of titanium available in a large energy range.

Transitions in ${ }^{56} \mathrm{Fe}\left(E_{\gamma}=847 \mathrm{keV}\right)$ and ${ }^{52} \mathrm{Cr}\left(E_{\gamma}=\right.$ $1434 \mathrm{keV}$ ) were frequently used as references but both of them have disadvantages. For the ${ }^{56} \mathrm{Fe}\left(n, n^{\prime} \gamma\right)$ reaction the main issues are the background contribution from components in the experimental setup, the nonisotropic angular distribution, and also the contributions from activation of the sample creating ${ }^{56} \mathrm{Mn}$, which via $\beta^{-}$decay populates ${ }^{56} \mathrm{Fe}$. $\operatorname{The}{ }^{52} \mathrm{Cr}\left(n, n^{\prime} \gamma\right)$ reaction has the same disadvantages with an additional problem represented by the difficulty in producing the sample $[5,6]$. These limitations indicate that other alternatives should be explored in order to obtain a more suitable reference cross section [7]. The best choices seem to be the 477.6-keV transition in ${ }^{7} \mathrm{Li}$ and the $983.5-\mathrm{keV}$ transition in ${ }^{48} \mathrm{Ti}$ [7-9].

The ${ }^{7} \mathrm{Li}$ transition was chosen because of its isotropic $\gamma$-ray emission, low inelastic threshold, negligible internal conversion coefficient and a reasonably smooth energy dependence of its cross section [10].

The transition in ${ }^{48} \mathrm{Ti}$ is also expected to have a reasonably high and smooth cross section over a large neutron energy range. The isotope has a relatively low production price and the sample can be easily prepared due to large availability of high-purity titanium. The disadvantages are related to angular anisotropy of the $\gamma$-ray emission and the possible contribution to the $983.5-\mathrm{keV} \gamma$ ray from the $\beta^{-}$decay of ${ }^{48} \mathrm{Sc}\left[T_{1 / 2}=\right.$ $43.67(9) \mathrm{h}]$, originating from the ${ }^{48} \mathrm{Ti}(n, p)$ reaction for $E_{n}>$ $5 \mathrm{MeV}$.

Previous inelastic neutron scattering experiments on $\mathrm{Ti}$ [11-21] with results available in the EXFOR database [22], are summarized in Table I. Even if the number of experiments is relatively large, most of them have covered a limited neutron-energy range. 
TABLE I. Summary of previous experiments on neutron inelastic scattering on titanium available in the EXFOR database [22]. The table also displays the year when the data was reported, the neutron producing reaction, the neutron energy range, the detectors used in the experiment, the detected particles, and the EXFOR entry.

\begin{tabular}{|c|c|c|c|c|c|}
\hline Reference & Year & $\begin{array}{l}\text { Neutron } \\
\text { production }\end{array}$ & $\begin{array}{c}E_{n} \text { range } \\
(\mathrm{MeV})\end{array}$ & Detectors & EXFOR entry \\
\hline D.L. Broder et al. [11] & 1965 & $\begin{array}{l}{ }^{3} \mathrm{H}(p, n){ }^{3} \mathrm{He} \\
{ }^{2} \mathrm{H}(d, n){ }^{3} \mathrm{He} \\
{ }^{3} \mathrm{H}(d, n){ }^{4} \mathrm{He}\end{array}$ & $1.1-3.2$ & Scintillator, $\gamma$ & 40035011 \\
\hline M.W. Pasechnik et al. [12] & 1969 & ${ }^{2} \mathrm{H}(d, n){ }^{3} \mathrm{He}$ & 2.9 & Scintillator, $n$ & 40045006 \\
\hline W. Breunlich et al. [13] & 1972 & ${ }^{3} \mathrm{H}(d, n){ }^{4} \mathrm{He}$ & 14.4 & $\mathrm{GeLi}, \gamma$ & 21286 \\
\hline W.E. Kinney [15] & 1973 & ${ }^{2} \mathrm{H}(d, n){ }^{3} \mathrm{He}$ & $4.07-8.56$ & Scintillator, $n$ & 10285019 \\
\hline E. Barnard [16] & 1974 & (unavailable) & $1.277-1.487$ & Scintillator, $n$ & 10048086 \\
\hline I.A. Korzh [17] & 1977 & ${ }^{3} \mathrm{H}(p, n){ }^{3} \mathrm{He}$ & $1.5-3.0$ & Scintillator, $n$ & 40532016 \\
\hline A.B. Smith [18] & 1978 & ${ }^{2} \mathrm{H}(d, n){ }^{3} \mathrm{He}$ & $1.5-4.5$ & Scintillator, $n$ & 13689004 \\
\hline A.I. Lashuk [19] & 1994 & $\begin{array}{l}{ }^{3} \mathrm{H}(p, n){ }^{3} \mathrm{He} \\
{ }^{2} \mathrm{H}(d, n){ }^{3} \mathrm{He} \\
{ }^{3} \mathrm{H}(d, n){ }^{4} \mathrm{He}\end{array}$ & $0.9-7.36,15-16$ & $\mathrm{GeLi}, \gamma$ & 41186007 \\
\hline
\end{tabular}

\section{EXPERIMENTAL PARTICULARITIES}

The present experiment was performed at EC-JRC Geel, Belgium, using the GELINA (Geel Electron Linear Accelerator) pulsed white neutron source [23-25] and the GAINS (Gamma Array for Inelastic Neutron Scattering) spectrometer [26,27]. The experimental setup and the data analysis procedure are described extensively in Refs. [24,28,29].

GELINA was running at $800 \mathrm{~Hz}$, and GAINS was placed on a measurement cabin located $200 \mathrm{~m}$ from the neutron source. Twelve detectors are positioned at $110^{\circ}, 150^{\circ}$, and $125^{\circ}$ with respect to the beam direction (four at each angle) at distances around $17 \mathrm{~cm}$ from the center of the sample. These detectors have $100 \%$ relative efficiency, and a typical $\gamma$-energy resolution of $2.8 \mathrm{keV}$ for the $1332-\mathrm{keV}$ peak of ${ }^{60} \mathrm{Co}$. The natural titanium disk that constituted the sample had the following characteristics: diameter $8.000(1) \mathrm{cm}$, thickness $0.45(1) \mathrm{cm}$ [i.e., $2.139(1) \mathrm{g} / \mathrm{cm}^{2}$ ] and purity $99.995 \%$. The areal densities corresponding to each of the five isotopes were determined using the isotopic composition of natural titanium [30], the values being displayed in Table II. The total irradiation time was $430 \mathrm{~h}$ corresponding to a statistical uncertainty of less than $0.5 \%$ for most of the observed $\gamma$-ray peaks (integrated over all neutron energies). In order to normalize the data, ${ }^{235} \mathrm{U}$ fission chamber with an efficiency of $86(4) \%$ was placed upstream of the titanium sample. The data acquisition system consisted of six ACQIRIS digitizers $\left(420 \times 10^{6}\right.$ samples/s and 12-bit amplitude resolution), with maximum two inputs and a common trigger, which recorded the waveforms from the HPGe preamplifiers and transferred them to a PC for further processing. A digital constant fraction algorithm was used to calculate the time of each signal. The amplitude was determined using an algorithm consisting of a correction for the decay constant of the preamplifier and for the ballistic deficit followed by an averaging of the signal before and after the event. The time information was used to calculate the incident neutron energy while the $\gamma$ energy was determined using the amplitude of the events. We obtained the yields of the observed transitions of interest and the yield of the fission chamber which were the starting points of the cross-section determination. The efficiencies of the detectors were determined using a ${ }^{152} \mathrm{Eu}$ point-like source and Monte Carlo simulations to take into account the effect of the extended sample. Additionally, Monte Carlo simulations were used also to correct the data for multiple scattering of neutrons in the sample and in the close vicinity of it.

Natural titanium has five stable isotopes with consecutive mass numbers. The incident neutron energy range was sufficient to open the $(n, 2 n \gamma)$ channel on several titanium isotopes (see Table III). This resulted in a contribution to the $\gamma$-production cross sections of each ${ }^{A} \mathrm{Ti}$ isotope from the ${ }^{A+1} \operatorname{Ti}(n, 2 n \gamma){ }^{A} \mathrm{Ti}$ reaction. We mention that it was impossible to extract the experimental $(n, 2 n \gamma)$ contributions, but these are taken into account when comparing our results with model calculations (see Sec. III).

\section{THEORETICAL CONSIDERATIONS}

Besides the comparisons with previous experimental values, our results are also compared with theoretical calculations performed with the TALYS 1.8 code [31]. These were done using

TABLE II. The isotopic abundance [30], and corresponding areal density of each stable isotope of the ${ }^{\text {nat }}$ Ti sample.

\begin{tabular}{|c|c|c|c|c|c|}
\hline Isotopes & ${ }^{46} \mathrm{Ti}$ & ${ }^{47} \mathrm{Ti}$ & ${ }^{48} \mathrm{Ti}$ & ${ }^{49} \mathrm{Ti}$ & ${ }^{50} \mathrm{Ti}$ \\
\hline Isotopic composition $(\%)$ & $8.25(3)$ & $7.44(2)$ & $73.72(3)$ & $5.41(2)$ & $5.18(2)$ \\
\hline Corresponding areal density $\left(\mathrm{g} / \mathrm{cm}^{2}\right)$ & $0.176(1)$ & $0.159(1)$ & $1.576(1)$ & $0.115(1)$ & $0.110(1)$ \\
\hline
\end{tabular}


TABLE III. The threshold energies for the $(n, 2 n \gamma)$ reactions of interest with the excitation energy of the first level included.

\begin{tabular}{|c|c|c|c|c|}
\hline Reaction & ${ }^{47} \mathrm{Ti}(n, 2 n \gamma){ }^{46} \mathrm{Ti}$ & ${ }^{48} \mathrm{Ti}(n, 2 n \gamma)^{47} \mathrm{Ti}$ & ${ }^{49} \mathrm{Ti}(n, 2 n \gamma){ }^{48} \mathrm{Ti}$ & ${ }^{50} \mathrm{Ti}(n, 2 n \gamma){ }^{49} \mathrm{Ti}$ \\
\hline$Q$ value $(\mathrm{MeV})$ & $9.0710(5)$ & $11.8710(5)$ & $9.313(5)$ & $11.1600(5)$ \\
\hline
\end{tabular}

two approaches: the "TALYS default" and the "TALYS modified structure" calculations.

The "TALYS default" calculation involves the TALYS semiempirical model with parameters obtained from global optimizations. It uses the optical model potentials with the local and global parametrization of Koning and Delaroche [32]. The Gilbert and Cameron approach [33] is exploited in order to calculate the level densities. At low energies the "TALYS default" calculations use the constant temperature model while at high energies they use the back shifted Fermi gas model with one energy dependent level density parameter which accounts for the damped shell effect proposed by Ignatyuk et al. [34]. For the $E 1$ transitions the $\gamma$-ray strength functions are described using the generalized Lorentzian form of Kopecky and Uhl [35], while for the other transition types the Brink-Axel option [36,37] is used. The de-excitation of the first 20 excited levels in the target and residual nuclei is described relying on a nuclear structure and decay table that is derived from the Reference Input Parameter Library (RIPL-3) [38]. The values are derived from the ones available in the ENSDF database [39]. When there are cases where the spin, parity or $\gamma$-decay path are unknown, TALYS assigns values based on simple statistical spin rules [31]. Importantly, the ${ }^{48} \mathrm{Ti}$ level scheme has several levels without an associated spin, parity, or decay path. In the following, two such levels lying in the energy range of interest are discussed: level 4 with $E_{l v l}=2465.0(5) \mathrm{keV}$ and level 6 with $E_{l v l}=3062.0(5) \mathrm{keV}$ (see Fig. 1). The 2465.0-keV level was observed only in a proton inelastic scattering experiment using a $65-\mathrm{MeV}$ polarized beam [40] but due to the low statistics no spin and parity have been assigned. The 3062.0-keV level was also observed in the experiment described in Ref. [40] and in an electron inelastic scattering experiment on ${ }^{48} \mathrm{Ti}$ [41]. For this level $J^{\pi}=2^{+}$was assigned from a model-independent plane-wave Born approximation (PWBA) analysis of the angular distribution. For example, in the default calculations TALYS makes the following assumptions:

(i) Level 4 has $J^{\pi}=1^{+}$and it decays to ground state through a $\gamma$ ray with $E_{\gamma}=2465.0 \mathrm{keV}$ and multipolarity $M 1$.

(ii) Level 6 decays through two $\gamma$ rays $\left[E_{\gamma}=2078.5 \mathrm{keV}\right.$ $(E 2)$ and $\left.E_{\gamma}=3062.0 \mathrm{keV}(M 1+E 2)\right]$ with equal branching ratios, to the first excited level and to the ground state, respectively.

The experimental spectra were investigated and no $\gamma$ rays were observed at the above mentioned energies even though TALYS estimates the cross section for the $2465.0 \mathrm{keV} \gamma$ ray to be comparable to the cross section of the $1437.5-\mathrm{keV}$ transition that was indeed observed with a maximum value of $0.250(12) \mathrm{b}$. The same comparison was performed for the two $\gamma$ rays emitted by the sixth level with the $2013.7-\mathrm{keV}$ transition because TALYS predicts similar values of the cross section for the three $\gamma$ rays. The 2013.7-keV cross section was experimentally observed with a value of $0.054(3) \mathrm{b}$ while the ones assumed by TALYS remained unobserved. These two investigations show that these $\gamma$ rays from the TALYS default calculations do not exist, or if they do, they are too weak to be experimentally observed; this is also consistent with the data reported in Ref. [20]. Finally, we examined the other possible de-excitation paths to the levels shown in Fig. 1, but no $\gamma$ rays of suitable energies were observed.

The "TALYS modified structure" calculations were performed with an input file of ${ }^{48} \mathrm{Ti}$ level scheme from which we deleted the two levels discussed above. The results of this calculation are also used for the comparison with the experimental values discussed in Sec. IV.

The " $\left(n, n^{\prime} \gamma\right)+(n, 2 n \gamma)$ " label refers to the sum of the two contributions predicted by the TALYS 1.8 code using the default input parameters. The theoretical predictions of the $(n, 2 n \gamma)$ cross sections are scaled with the abundance of each stable isotope.

\section{RESULTS AND DISCUSSION}

This section will present and discuss the experimental results of the ${ }^{\text {nat }} \operatorname{Ti}\left(n, n^{\prime} \gamma\right)$ reaction and is structured in two

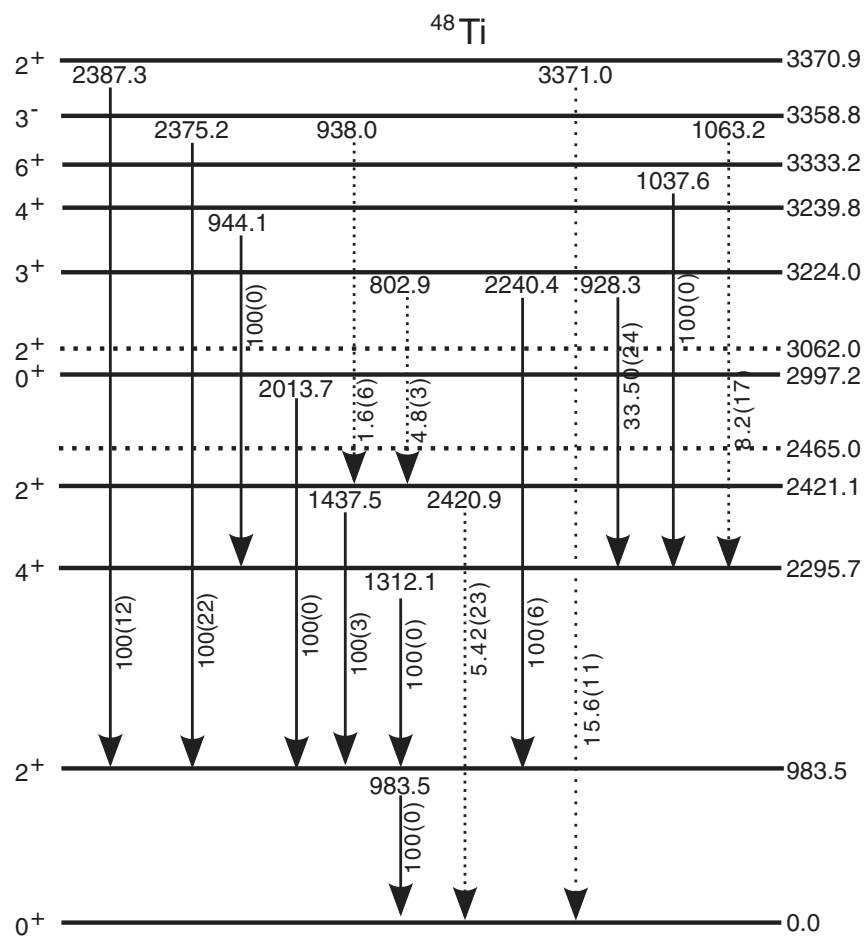

FIG. 1. Simplified level scheme of the ${ }^{48} \mathrm{Ti}$ nucleus [39]. The observed transitions are displayed using solid lines. The dashed line is used to show known transitions that were not observed but were taken into account in the data analysis procedure or to display the levels with no or unknown $\gamma$-ray contribution. 


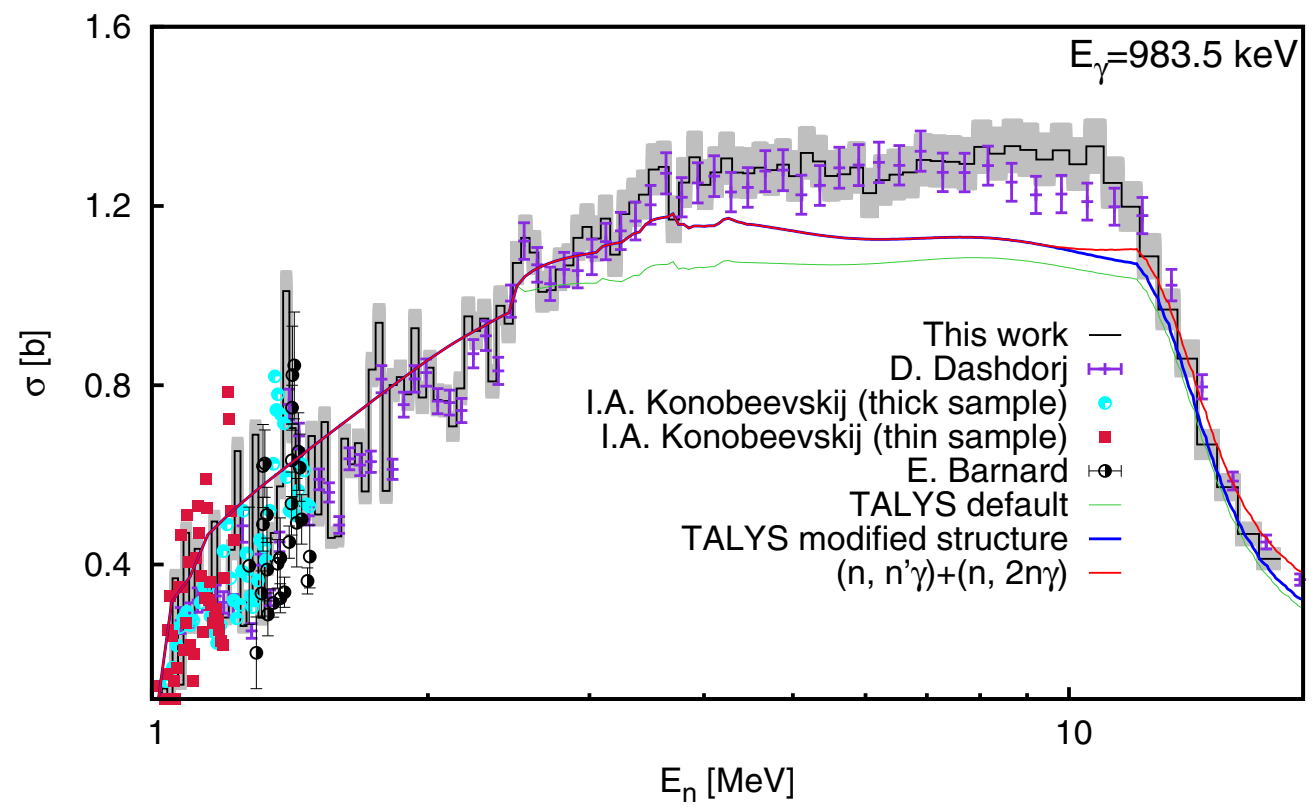

FIG. 2. The $\gamma$ production cross section of the first transition in ${ }^{48} \mathrm{Ti}$ in comparison with previous experimental results and with TALYS 1.8 theoretical calculations. The gray band represents the total absolute uncertainties of our experimental values.

subsections. We will start with the results for the ${ }^{48} \operatorname{Ti}\left(n, n^{\prime} \gamma\right)$ reaction followed by the neutron inelastic scattering reactions on the minor stable isotopes of ${ }^{\text {nat }} \mathrm{Ti}\left({ }^{46,47,49,50} \mathrm{Ti}\right)$. All of them will be discussed in terms of $\gamma$-production cross section, level cross sections, and total inelastic cross sections. The $\gamma$-production cross sections of each nucleus are the primary experimental results. These are further used to determine the level cross sections based on the feeding and the decay of each level. The total inelastic cross sections are calculated as the sum of the cross sections of all the observed $\gamma$ rays that populate the ground state. To reduce the statistical uncertainties, several time-of-flight bins were combined, with a resulting reduction of the neutron energy resolution.

\section{A. Neutron inelastic scattering on ${ }^{48} \mathrm{Ti}$}

\section{1. $\gamma$-production cross sections}

From the inelastic scattering of neutrons on the most abundant isotope of titanium, ${ }^{48} \mathrm{Ti}(73.72 \%)$, we identified ten $\gamma$ rays of interest (see Fig. 1). For each observed $\gamma$ ray we calculated the integrated $\gamma$-production cross section and the results are compared in Figs. 2 and 3 with TALYS 1.8 calculations and with other experimental data. For the first transition (Fig. 2), the comparison between our results and the values reported by Dashdorj et al. shows a fairly good agreement except for a small (1-2 $\sigma$ ) difference between 8 and $10 \mathrm{MeV}$. Both experiments have a large number of data points with low uncertainty, especially for the first $\gamma$ ray.

A notable difference between our results and those of Ref. [20] is visible in Fig. 3(e) $\left(E_{\gamma}=928.3 \mathrm{keV}\right)$ where our values are roughly twice higher. In order to understand the difference we have also to look at panel (d) $\left(E_{\gamma}=\right.$ $2240.4 \mathrm{keV})$. Both transitions de-excite the same level $\left(E_{l v l}=\right.$ $3224.0 \mathrm{keV}$ ) which means that the shape of the cross section is the same for both $\gamma$ rays. The branching ratios, of the above two transitions, used in the TALYS calculations are the currently accepted literature values based on available experimental data and are the following: $I_{\gamma}(2240.4 \mathrm{keV})=100 \%$ and $I_{\gamma}(928.3 \mathrm{keV})=33.46(24) \%$ [39]. Using our experimental results we determined the relative intensity for the $928-\mathrm{keV}$ transition by simply dividing the cross sections of the two transitions and the value we got $[35.52(4) \%]$ is close to the one available in the literature. On the other hand, we performed the same calculation using Dashdorj's cross section values and his relative intensity was $19.81(30) \%$, which is approximately half of our value. This is consistent with the difference between our values and the values reported in Ref. [20] and raises a question mark on the data reported by Dashdorj et al. for this $\gamma$ ray.

Differences between the experimental data sets can be also observed in Figs. 3(a)-3(d), 3(f), 3(h). Our data have consistently higher values than that from Ref. [20] between 8-12 MeV neutron energy. These cannot be related to the $(n, 2 n \gamma)$ contributions because they start below the reaction threshold. Although the uncertainty of our data is larger in this range, the difference is clear and systematic and points to an instrumental or setup related effect.

Figures 2 and 3 display also the comparison between the experimental integrated $\gamma$-production cross sections and the theoretical ones calculated using the TALYS 1.8 code as described in Sec. III [see Figs. 2 and 3(b)-3(e)]. We note that all the theoretical calculations underestimate the experimental results except for low energies. The contribution coming from the ${ }^{49} \mathrm{Ti}(n, 2 n \gamma)^{48} \mathrm{Ti}$ is almost insignificant. This was expected because of the large difference between the isotopic abundances of ${ }^{48} \mathrm{Ti}$ and ${ }^{49} \mathrm{Ti}$.

It is particularly interesting to note that the agreement among the experimental values and the TALYS results was improved by performing the "TALYS modified structure" 


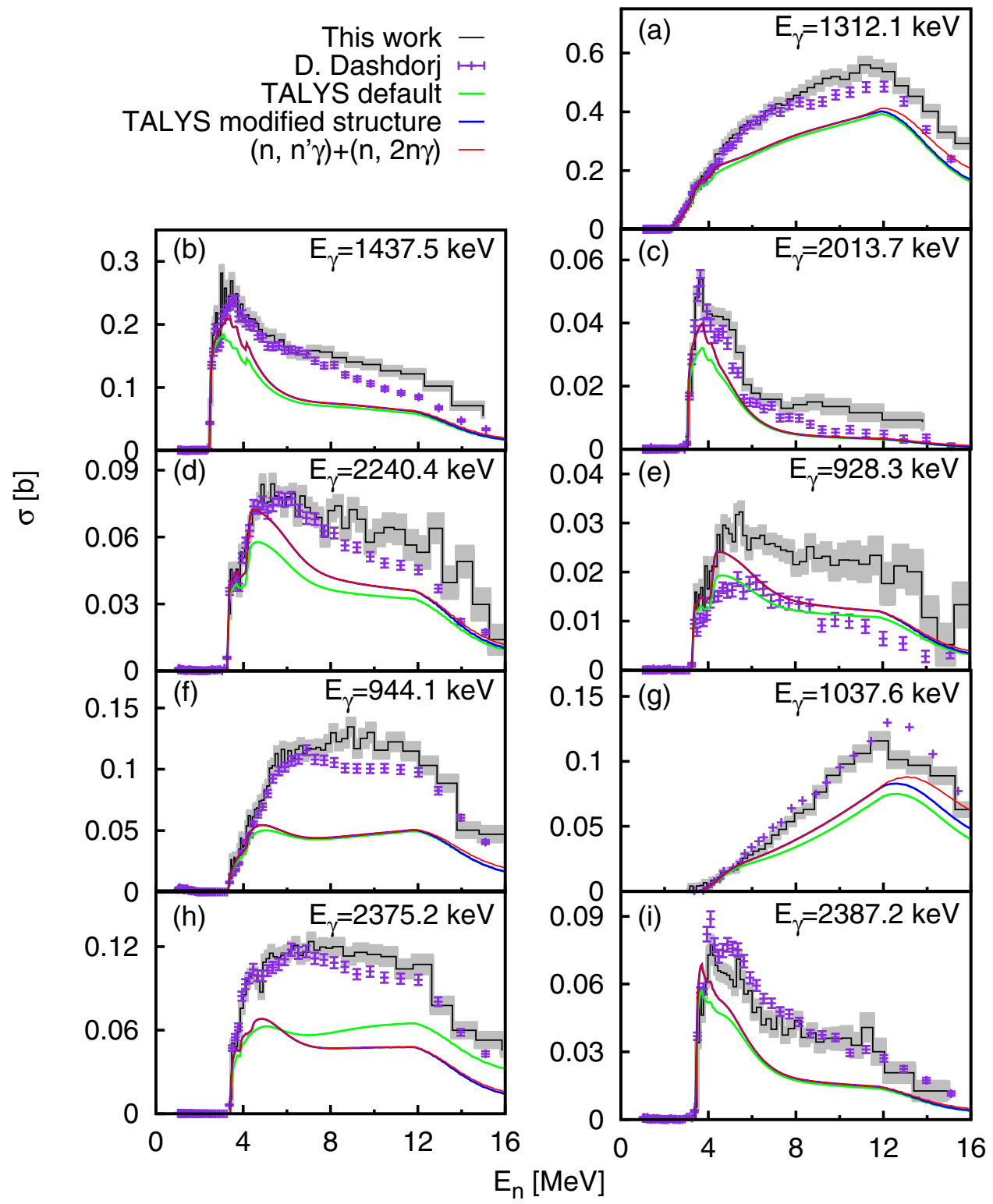

FIG. 3. The $\gamma$ production cross section for the other transitions observed in ${ }^{48} \mathrm{Ti}$ in comparison with previous experimental reported results and with TALYS 1.8 theoretical calculations. The total absolute uncertainties of our values are displayed in gray.

TABLE IV. The formulas used to calculate the level cross sections for the excited states in ${ }^{48} \mathrm{Ti}$, the weights of the observed $\gamma$ rays and the neutron energy ranges where they apply.

\begin{tabular}{lcc}
\hline \hline Level $(\mathrm{keV})$ & Formula & Range $(\mathrm{keV})$ \\
\hline 983.5 (This work) & $\sigma_{983.5}^{\gamma}\left(E_{n}\right)-\sigma_{1312.1}^{\gamma}\left(E_{n}\right)-\sigma_{1437.5}^{\gamma}\left(E_{n}\right)-\sigma_{2013.7}^{\gamma}\left(E_{n}\right)-\sigma_{2240.4}^{\gamma}\left(E_{n}\right)-\sigma_{2375.2}^{\gamma}\left(E_{n}\right)-\sigma_{2387.2}^{\gamma}\left(E_{n}\right)$ & $1004.2-3692.9$ \\
2295.7 (This work) & $\sigma_{1312.1}^{\gamma}\left(E_{n}\right)-\sigma_{928.3}^{\gamma}\left(E_{n}\right)-\sigma_{944.1}^{\gamma}\left(E_{n}\right)-\sigma_{1037.6}^{\gamma}\left(E_{n}\right)-0.082 \sigma_{2375.2}^{\gamma}\left(E_{n}\right)$ & $2343.9-3582.4$ \\
2421.1 (This work) & $1.0542 \sigma_{1437.5}^{\gamma}\left(E_{n}\right)-0.048 \sigma_{2240.4}^{\gamma}\left(E_{n}\right)-0.016 \sigma_{2375.2}^{\gamma}\left(E_{n}\right)$ & $2472.0-3692.9$ \\
983.5 (D. Dashdorj) & $\sigma_{983.5}^{\gamma}\left(E_{n}\right)-\sigma_{1312.1}^{\gamma}\left(E_{n}\right)-\sigma_{1437.5}^{\gamma}\left(E_{n}\right)-\sigma_{2013.7}^{\gamma}\left(E_{n}\right)-\sigma_{2240.4}^{\gamma}\left(E_{n}\right)-\sigma_{2375.2}^{\gamma}\left(E_{n}\right)-\sigma_{2387.2}^{\gamma}\left(E_{n}\right)-$ & $1004.2-4892.8$ \\
& $-\sigma_{2633.2}^{\gamma}\left(E_{n}\right)-\sigma_{2715.8}^{\gamma}\left(E_{n}\right)-\sigma_{2756.0}^{\gamma}\left(E_{n}\right)-\sigma_{2819.1}^{\gamma}\left(E_{n}\right)-\sigma_{2868.6}^{\gamma}\left(E_{n}\right)-\sigma_{3090.8}^{\gamma}\left(E_{n}\right)-\sigma_{3328.0}^{\gamma}\left(E_{n}\right)-$ & $2343.9-4676.1$ \\
2295.7 (D. Dashdorj) & $-\sigma_{3403.8}^{\gamma}\left(E_{n}\right)-\sigma_{3473.9}^{\gamma}\left(E_{n}\right)-\sigma_{3596.7}^{\gamma}\left(E_{n}\right)-0.20 \sigma_{1140.9}^{\gamma}\left(E_{n}\right)-\sigma_{3808.6}^{\gamma}\left(E_{n}\right)$ & \\
& $\sigma_{1312.1}^{\gamma}\left(E_{n}\right)-\sigma_{928.3}^{\gamma}\left(E_{n}\right)-\sigma_{944.1}^{\gamma}\left(E_{n}\right)-\sigma_{1037.6}^{\gamma}\left(E_{n}\right)-0.082 \sigma_{2375.2}^{\gamma}\left(E_{n}\right)-\sigma_{1212.8}^{\gamma}\left(E_{n}\right)-\sigma_{2086.0}^{\gamma}\left(E_{n}\right)-$ & \\
2421.1 (D. Dashdorj) & $-0.85 \sigma_{3403.8}^{\gamma}\left(E_{n}\right)-\sigma_{2109.0}^{\gamma}\left(E_{n}\right)-\sigma_{2161.8}^{\gamma}\left(E_{n}\right)-\sigma_{2285.4}^{\gamma}\left(E_{n}\right)$ & $2472.0-4472.3$ \\
& $\sigma_{1437.5}^{\gamma}\left(E_{n}\right)+\sigma_{2420.9}^{\gamma}\left(E_{n}\right)-0.048 \sigma_{2240.4}^{\gamma}\left(E_{n}\right)-0.016 \sigma_{2375.2}^{\gamma}\left(E_{n}\right)$ & \\
\hline \hline
\end{tabular}




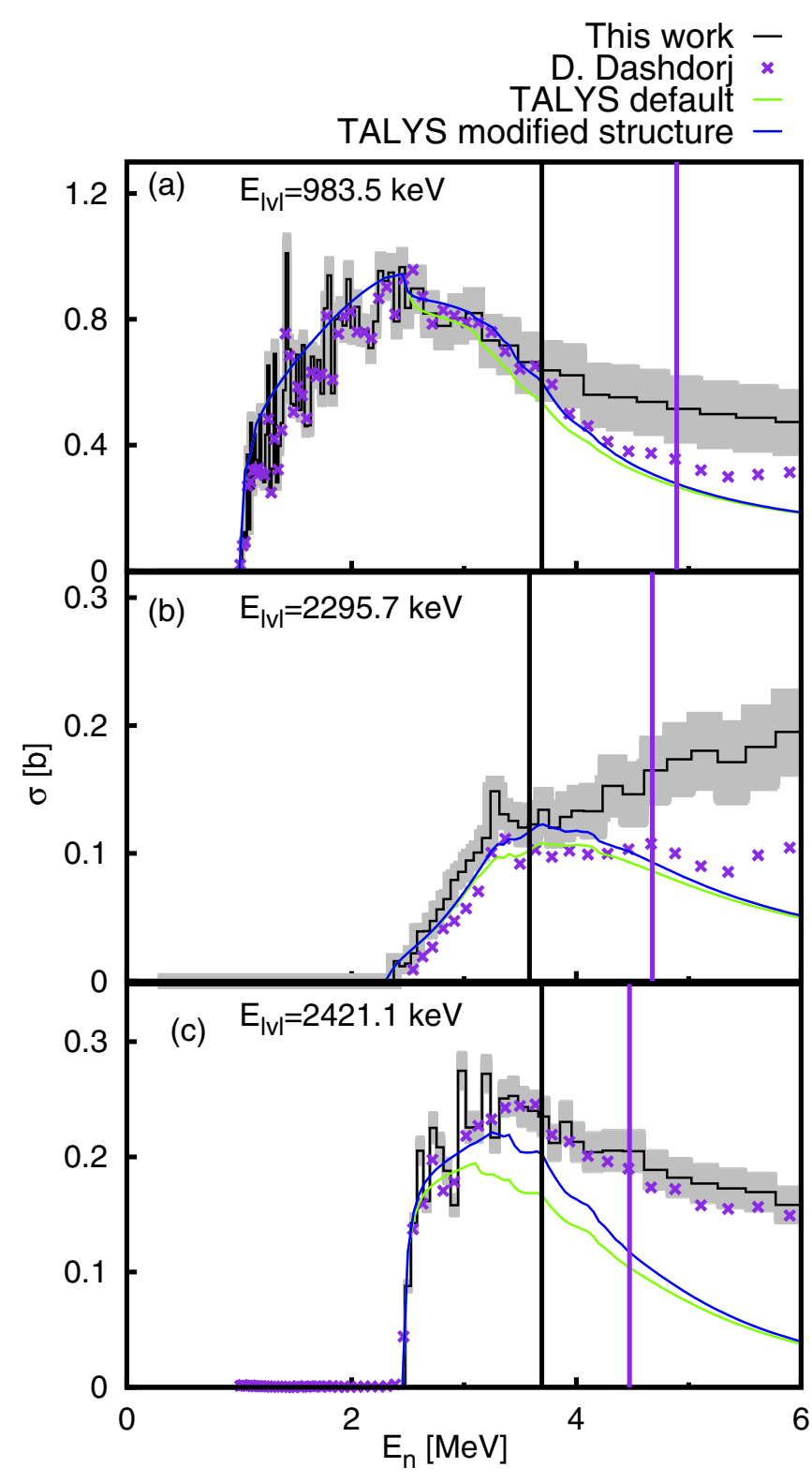

FIG. 4. Level cross sections in ${ }^{48} \mathrm{Ti}$ in comparison with the results reported by Dashdorj et al. [20] and TALYS 1.8 calculations. The experimental results are precise up to the higher limit of the range indicated by vertical lines (black for us and purple for the results from Ref. [20]). Above that value the results represent upper limits of the level cross section. The gray band constitutes the total absolute uncertainties of our experimental values.

calculations as explained in Sec. III. The remaining difference between TALYS and the experimental values is most likely associated with the specific level excitation and decay estimates, since the good agreement for the total inelastic cross section (see Figs. 5 and 8 ) validates the optical model.

\section{Level cross sections}

The level cross sections were determined using the values of the integrated $\gamma$-production cross sections taking into account the feeding and the decay of each level of interest. The feeding from higher states is subtracted from the level cross sections, weighted by the relative intensities of the $\gamma$ rays. The results are valid up to the inelastic threshold of the first level having an undetected transition feeding the level of interest. Above it the level cross section is only the upper limit. Table IV displays the formulas used to determine the level cross sections and the validity range. Both Table IV and Fig. 4 present only the cases for which we also observed $\gamma$ rays which populate the levels of interest. Otherwise, the resulting level cross sections would be identical to the production cross section of the $\gamma$ rays de-exciting the level. We also calculated the level cross sections from the data by Dashdorj et al. using the equations shown in Table IV and the level information from Ref. [39]. The cross sections reported by Dashdorj et al. are valid to higher energies as more $\gamma$ rays were observed (see Table IV). Figure 4 shows the comparison between the level cross sections up to $E_{n}=$ $6 \mathrm{MeV}$, which is above the accuracy limits. A good agreement exists between all the results in the specified validity range.

\section{Total inelastic cross section}

The total inelastic cross section was calculated as a sum of $\gamma$-production cross sections of all the detected transitions that feed the ground state. The same type of calculation was performed also using the data reported by Dashdorj in EXFOR. The formulas used to determine the total inelastic cross sections are displayed in Table V. The values are precise in a neutron energy range where the limits of the range are defined as for the level cross sections; beyond the upper limit, our values are only lower limits. The total inelastic cross section is compared in Fig. 5 with TALYS 1.8 calculations and evaluated nuclear data libraries ENDFB/B-VII.1 [42] and JEFF-3.2 [43]. The total inelastic cross section on ${ }^{48} \mathrm{Ti}$ reaches the highest value around $1.30(5) \mathrm{b}$ at $E_{n} \approx 4 \mathrm{MeV}$. The agreement between our data, the results of Dashdorj et al. and the theoretical calculations is very good. Both experimental data sets agree nicely despite differences in the number of $\gamma$ rays underlying the estimated total inelastic cross section and the resulting difference in maximum energy. The difference of $1-2 \sigma$ in the $8-10 \mathrm{MeV}$ incident energy range is observed also here. The conclusion from the comparison with the model calculation is that the higher lying levels do not show significant decay branches to the ground state, but rather mostly decay to the ground state via the levels for which

TABLE V. The formulas used to calculate the total inelastic cross section for ${ }^{48} \mathrm{Ti}$ and the neutron energy ranges where they apply.

\begin{tabular}{lcc}
\hline \hline Level $(\mathrm{keV})$ & Formula & Range $(\mathrm{keV})$ \\
\hline g.s. (This work) & $\sigma_{983.5}^{\gamma}\left(E_{n}\right)+0.054 \sigma_{1437.5}^{\gamma}\left(E_{n}\right)+0.156 \sigma_{2387.2}^{\gamma}\left(E_{n}\right)$ & $1004.2-3692.9$ \\
g.s. (D. Dashdorj) & $\sigma_{983.5}^{\gamma}\left(E_{n}\right)+\sigma_{2420.9}^{\gamma}\left(E_{n}\right)+\sigma_{3371.0}^{\gamma}\left(E_{n}\right)+0.014 \sigma_{2633.2}^{\gamma}\left(E_{n}\right)$ & $1004.2-4293.8$ \\
& $+\sigma_{3699.1}^{\gamma}\left(E_{n}\right)+\sigma_{3738.4}^{\gamma}\left(E_{n}\right)+0.16 \sigma_{3090.8}^{\gamma}\left(E_{n}\right)+0.64 \sigma_{972.9}^{\gamma}\left(E_{n}\right)$ & \\
\hline \hline
\end{tabular}




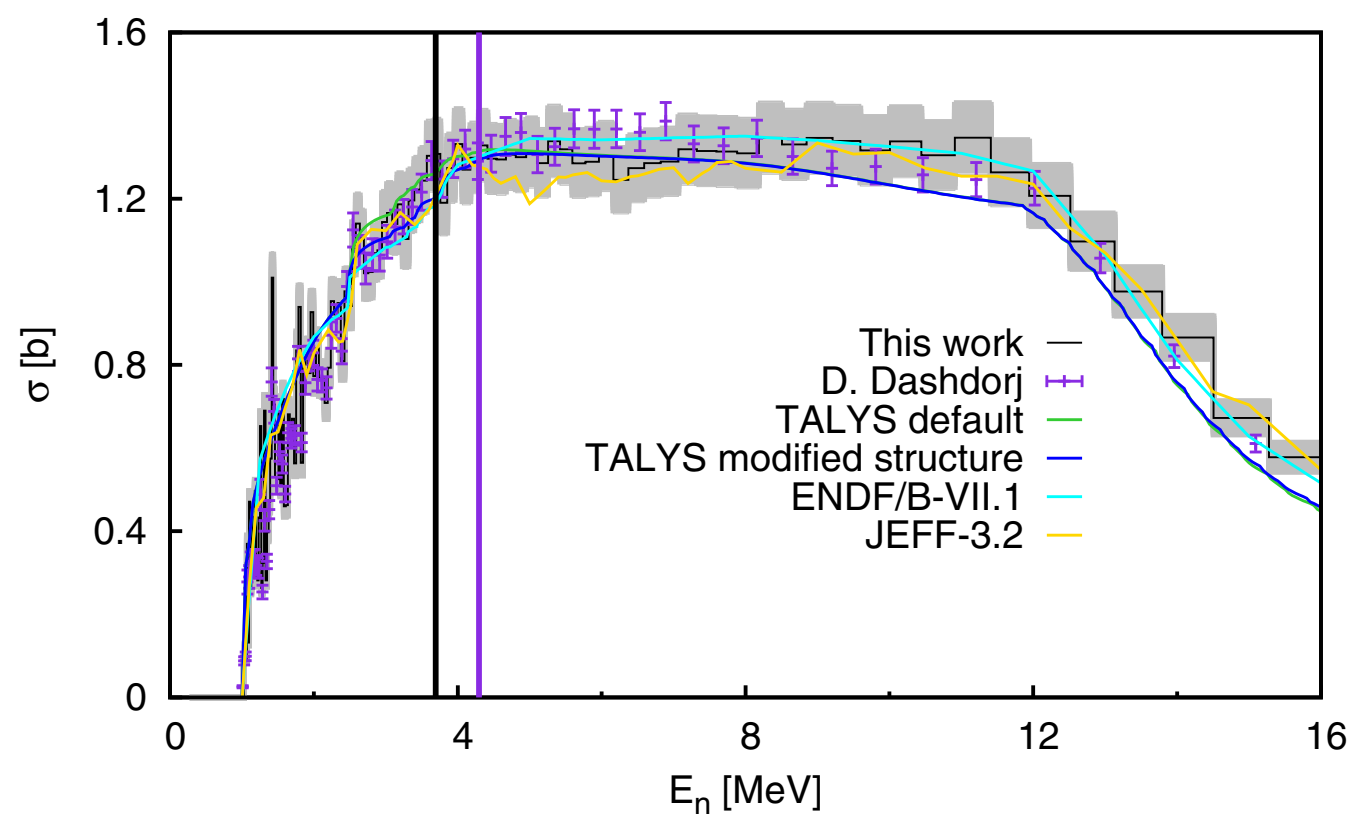

FIG. 5. The experimental total inelastic cross section for ${ }^{48} \mathrm{Ti}$ in comparison with the data reported by Dashdorj et al., TALYS theoretical calculations and evaluated nuclear data libraries ENDF/B-VII.1 and JEFF-3.2. The vertical lines represent the upper limit of the range in which the total inelastic cross section is accurate. The black line corresponds to our results while the purple one to the results reported in Ref. [20]). The gray band displays the total absolute uncertainties.

we and Dashdorj observed the decay. Of course the TALYS inelastic cross section is not based on the decay calculation but rather comes directly from the optical model (especially above $4 \mathrm{MeV}$ where compound elastic scattering is negligible). The nice agreement with TALYS validates the default optical model used in the theoretical calculations.

\section{B. Neutron inelastic scattering on ${ }^{46,47,49,50} \mathrm{Ti}$}

Because of using a natural titanium sample, we observed transitions (see Fig. 6) also from the minor isotopes of titanium $\left({ }^{46,47,49,50} \mathrm{Ti}\right)$. For each of these $\gamma$ rays we calculated the integrated $\gamma$-production cross sections, and based on them the total inelastic cross sections, where possible. The level cross sections were not determined because of not observing the feeding $\gamma$ rays from higher levels. In these cases the resulting level cross sections would be identical to the production cross section of the $\gamma$ rays de-exciting the level.

\section{1. $\gamma$-production cross sections}

The transitions for which we were able to determine the $\gamma$-production cross sections are displayed in Fig. 6 with solid arrows. The first transition in ${ }^{47} \mathrm{Ti}\left(E_{\gamma}=159.4 \mathrm{keV}\right)$ was not properly observed because the thresholds of our detection electronics were set too high during the experiment.

Figure 7 displays the $\gamma$ production cross sections of all $\gamma$ rays observed from the inelastic scattering of neutrons on the minor isotopes of nat $\mathrm{Ti}$. Our results are compared only with theoretical calculations performed using the TALYS 1.8 code as no other experimental data are available. Except for three cases [panels (a)-(c)] we limit the energy range up to $E_{n}=6 \mathrm{MeV}$. Indeed, the cross sections from panels (d)-(k) are rather small, and therefore, the uncertainties above $6 \mathrm{MeV}$ are too high to allow a meaningful comparison with theory.

Note that for ${ }^{50} \mathrm{Ti}$ the cross section of the first transition [Fig. 7(c)] is similarly underestimated as for ${ }^{48} \mathrm{Ti}$, while, in contrast, the first transitions cross section in ${ }^{46} \mathrm{Ti}$ is described well by the TALYS calculations. For the case of ${ }^{47} \mathrm{Ti}$ two of the $\gamma$-production cross sections $\left(E_{\gamma}=1284.9 \mathrm{keV}\right.$ and $E_{\gamma}=$ $1390.3 \mathrm{keV}$ ) are well described by theoretical calculations while the values for the other two are overestimated below $4 \mathrm{MeV}$. For ${ }^{49} \mathrm{Ti}$ the situation is reversed: two $\gamma$-production cross sections $\left(E_{\gamma}=1622.6 \mathrm{keV}\right.$ and $\left.E_{\gamma}=1762.0\right)$ are significantly underestimated by the TALYS while for the other three the agreement is good.

Overall, the comparison shows a good agreement between the experimental and theoretical values for most of the cross sections up to around $6 \mathrm{MeV}$ incident energy.

\section{Total inelastic cross sections}

The total inelastic cross sections were also determined, except for ${ }^{47} \mathrm{Ti}$. Figure 8 displays the experimental total inelastic cross section in comparison with theoretical calculations performed using TALYS 1.8 code with default input parameters, and the values from the evaluated databases ENDF/B-VII.1 [42], JEFF-3.2 [43], and CENDL-3.1 [48]. The comparison is performed up to $9 \mathrm{MeV}$ where the $(n, 2 n \gamma)$ reaction channels start to contribute. Even though for ${ }^{46} \mathrm{Ti}$ and ${ }^{50} \mathrm{Ti}$ only the first transition was observed, agreement between the experimental data, TALYS calculations, and the evaluated libraries is very good. This indicates that other $\gamma$ rays feeding the ground state have small contributions, which were calculated (using the cross sections of the observed and unobserved $\gamma$ rays from TALYS 1.8 ) to be $\approx 3 \%$. 


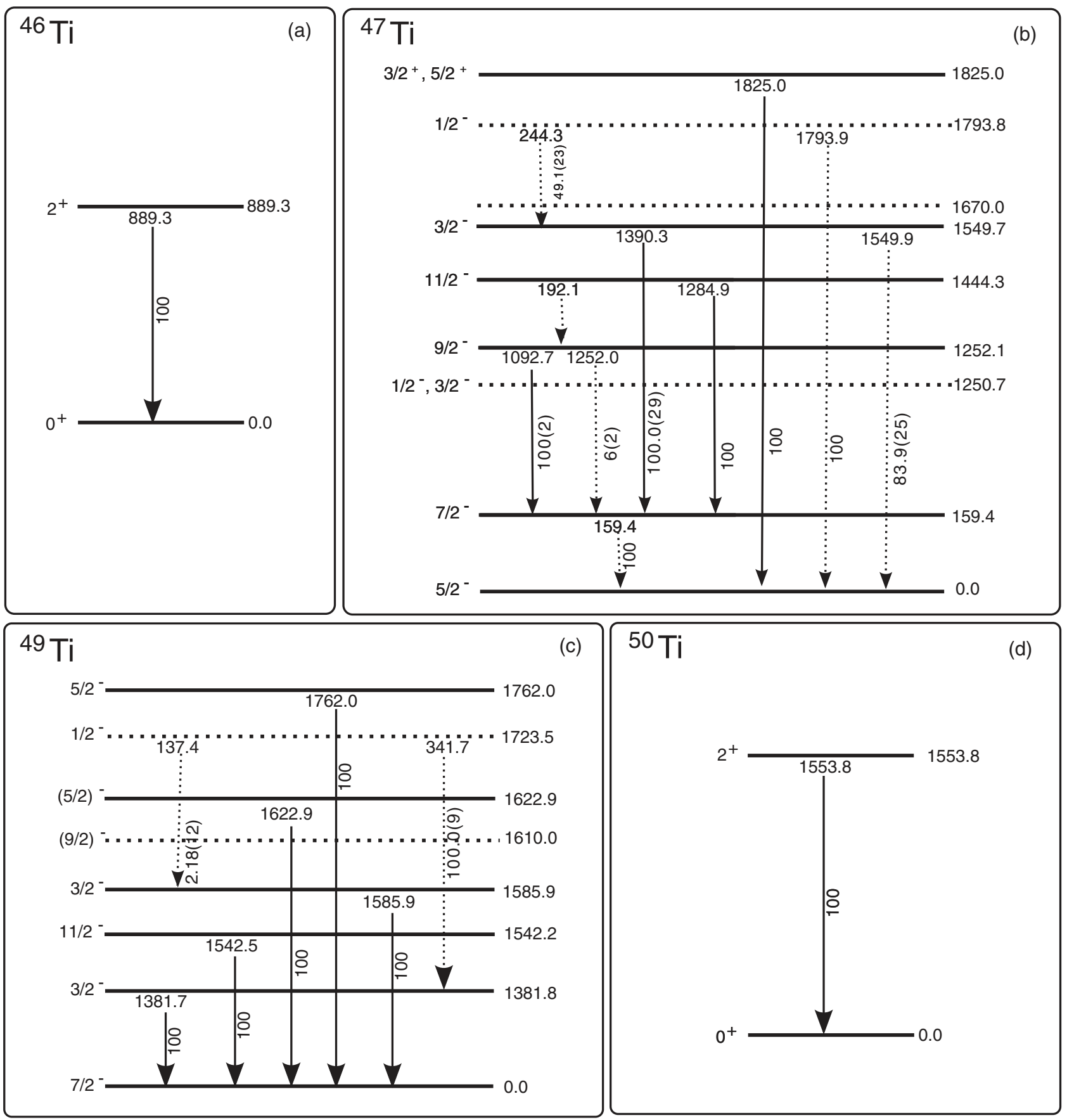

FIG. 6. Simplified level scheme of the minor isotopes of natural titanium [44-47]. The observed $\gamma$ rays are displayed with solid lines. The dashed lines are used to show known transitions that were not observed but were taken into account in the data analysis procedure.

For the case of ${ }^{49} \mathrm{Ti}$, all five detected $\gamma$ rays feed the ground state and the contribution coming from them can be observed in Fig. 8(c) where each step-like feature represents a $\gamma$ ray production cross section to the total inelastic cross section (see Fig. 6). Of course that, from a mathematical point of view, this is straightforward considering the formula used to determine the experimental total inelastic cross section. However, it is interesting to notice the fact that each step represents the excitation of a new, higher, individual level in the studied nucleus which then de-excites and contributes to the total inelastic cross section. The comparable cross sections of the five transitions mentioned above allow us to observe the opening of each low-lying level of the coupled channels.

A similar structure is observed also in ${ }^{46,50} \mathrm{Ti}$ although in these cases the feature is visible directly in the shape of the first $\gamma$ production cross sections. In all cases the theoretical calculated curves nicely reproduce the features.

\section{Uncertainties}

The total relative uncertainty for the $\gamma$ production cross section of the strongest transition in all the titanium isotopes 

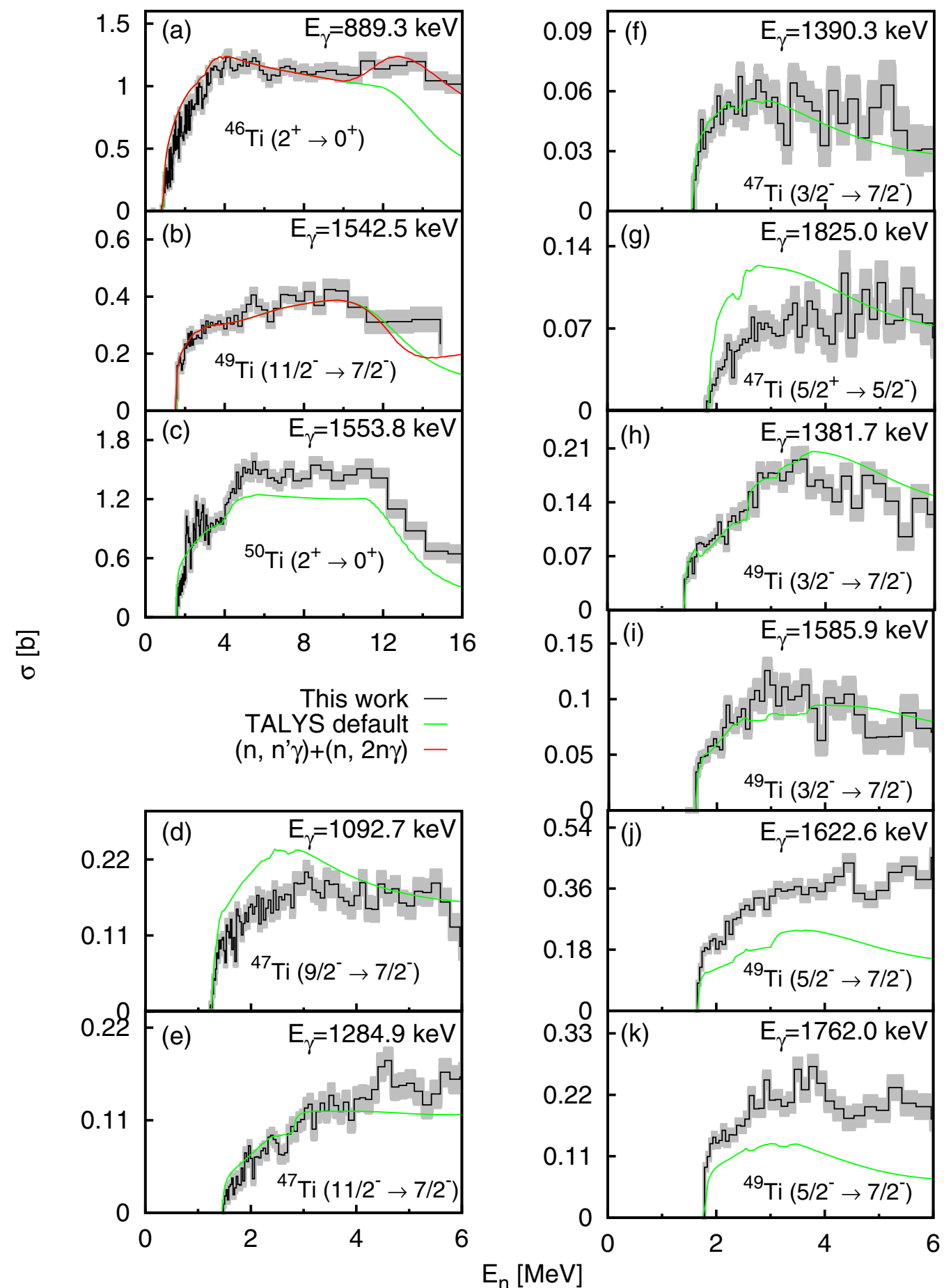

FIG. 7. The production cross sections of the $\gamma$ rays observed from the minor isotopes of ${ }^{\text {nat }} \mathrm{Ti}$ in comparison with TALYS theoretical calculations performed using the default input parameters. The total absolute uncertainties of our experimental values are displayed in gray.

is around 5\%, while for the weaker ones they are below $10 \%$ for all neutron energies. Indeed the uncertainties increase with the incident neutron energy as a result of a drop in the neutron flux. This is kept under control by rebinning the data in regions where the cross section is more or less structureless.

The main sources of uncertainty are the yields of the HPGe detectors $(2 \%)$ and of the fission chamber $(3 \%)$, and the detectors' efficiency calculations (2-4\%) mainly coming from the activity of the calibration source and from the detector-source geometry. The efficiency of the fission chamber contributes with a uncertainty 3-4\%. The MCNP simulations introduce an uncertainty of about $1 \%$ while the uncertainties of other physical quantities used in calculating the cross sections (e.g., sample dimensions, isotopic abundances, the fission cross section of ${ }^{235} \mathrm{U}$, etc.) are under $1 \%$. All these values were taken into account when calculating the total absolute uncertainties 


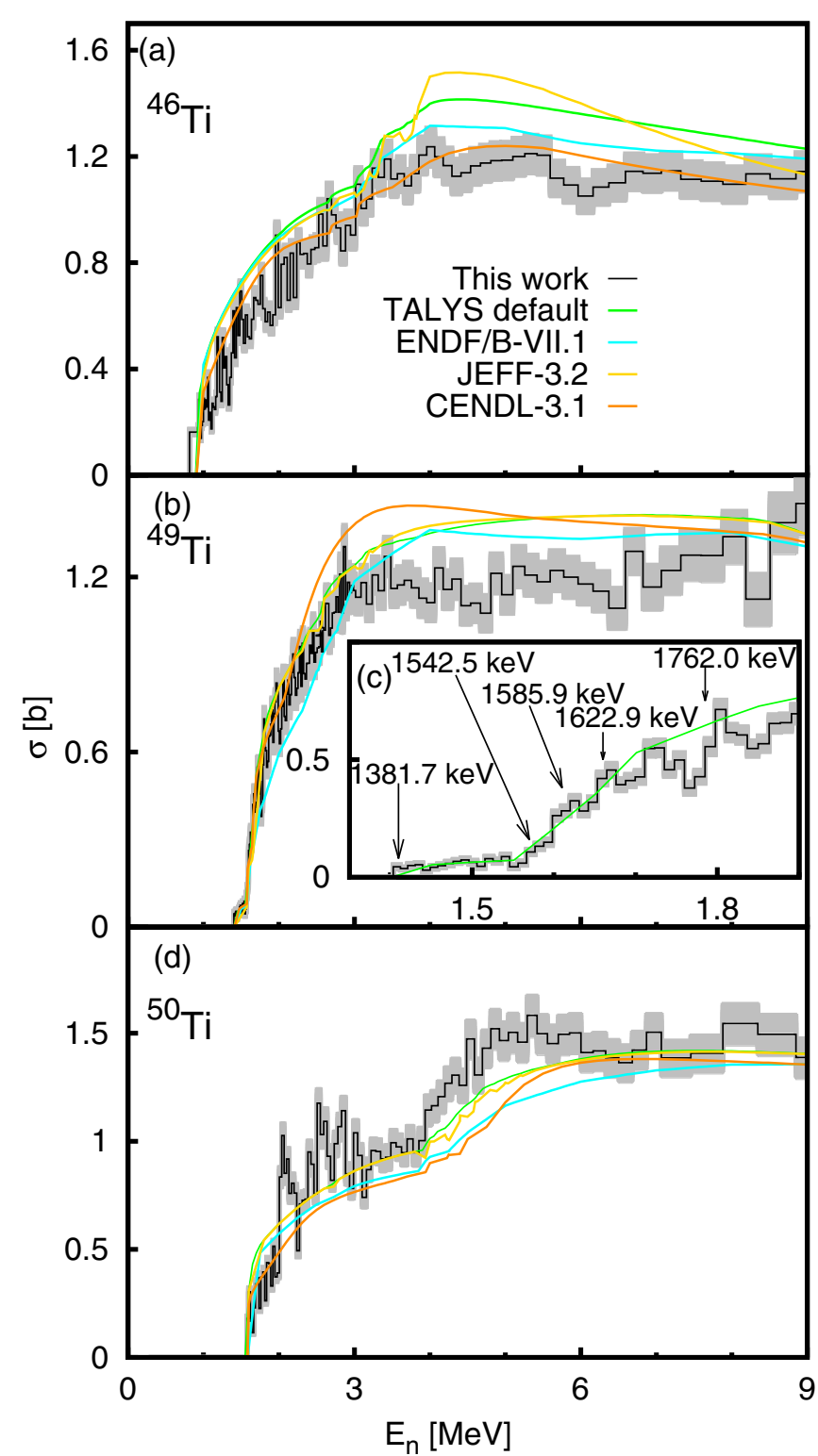

FIG. 8. The total inelastic cross sections of ${ }^{46,49,50} \mathrm{Ti}$ compared with TALYS calculations and values from the evaluated database ENDF/B-VII.1, and JEFF-3.2 and CENDL-3.1. (c) displays a zoom of the total inelastic cross section for ${ }^{49} \mathrm{Ti}$ with arrows indicating the contribution of each observed $\gamma$ ray. The total absolute uncertainties of the experimental values are shown in gray.

displayed with gray bands in the figures which present the experimental results.

\section{THE ${ }^{48} \operatorname{Ti}\left(n, n^{\prime} \gamma\right)$ CROSS SECTION AS A STANDARD}

This section deals with the possibility of using the $\gamma$ production cross section of the first transition in ${ }^{48} \mathrm{Ti}\left(E_{\gamma}=\right.$ $983.5 \mathrm{keV}$ ) as a reference for future measurements. The yield of the transition was already evaluated a few years ago taking into account all the available experimental results [6]. Up to that time only Dashdorj et al. provided values of the cross sections in the energy range from threshold up to $20 \mathrm{MeV}$.
In Ref. [6] it was concluded that a new, independent, more precise cross-section measurement was needed. This was one of the main motivations of the present experiment.

The comparison between our results and the one in Ref. [20] can be seen in Fig. 2 and shows an excellent agreement (i.e., $\chi^{2} \approx 0.04$ ), except for a few points between $E_{n}=8-10 \mathrm{MeV}$ where the agreement is within 1-2 $\sigma$. The fact that we had a natural titanium sample, whereas Dashdorj et al. used an enriched $(99.81 \%){ }^{48} \mathrm{Ti}$ sample [20], is a tempting possibility to explain the difference. However, as the discrepancy appears below the $(n, 2 n)$ threshold, it seems that this is not the reason. Another difference between the two experiments is the energy range of the incoming neutrons. Dashdorj had neutrons with $E_{n}$ up to $250 \mathrm{MeV}$ resulting in a complicated $\gamma$ spectrum due to contributions from other reaction channels (in total 11 different isotopes were created in that experiment) [20]. The neutron energy range available in our case was an advantage in terms of not opening many other reactions channels.

A $\gamma$-ray spectrum from one of the GAINS HPGe detectors, showing the region around the $983-\mathrm{keV}$ transition, is presented in Fig. 9. The region around the peak is free of any strong background component. The nearest peak is at $E_{\gamma}=968.9 \mathrm{keV}$ and is coming from the natural decay chain of ${ }^{228} \mathrm{Ac}$. However, the energy resolution of any typical HPGe detector is sufficient to separate the two peaks. Also its area (in our case) is only $3 \%$ of the area of the $983.5-\mathrm{keV} \gamma$ ray.

The agreement level for the rest of the incident energy range is rather impressive taking into account that the two experiments were performed at different facilities and using different samples. Of course similarities exist between the two experiments such as the method to determine the neutron energies (time of flight), the detection method ( $\gamma$ spectroscopy using HPGe detectors) and the use of a fission chamber to determine the neutron flux $\left({ }^{235} \mathrm{U}\right.$ in the present work and ${ }^{235,238} \mathrm{U}$ foils in Dashdorj's).

The cross section is indeed almost constant $(\approx 1.3 \mathrm{~b})$ for $E_{n} \approx 4-12 \mathrm{MeV}$. Our results have an uncertainty below $5 \%$ for the full neutron energy range, and a good neutron energy resolution, which allows us to report more than 100 experimental points for the cross section of the $983.5-\mathrm{keV}$ $\gamma$ ray.

All these arguments favor the use of the first transition in ${ }^{48} \mathrm{Ti}$ as a reference cross section. Our results were already made available to the working group performing a new evaluation of this nucleus with the purpose of developing a standard cross section.

\section{CONCLUSIONS}

The present article reports the results obtained in a neutron inelastic scattering experiment on a ${ }^{\text {nat }}$ Ti sample.

The experiment was performed at the GELINA white neutron source of the JRC-Geel facility using the GAINS spectrometer. We observed in total $21 \gamma$ rays following $\left(n, x n^{\prime} \gamma\right)$ reactions on $\left({ }^{46-50} \mathrm{Ti}\right)$. We determined the $\gamma$ production cross sections, the level cross sections, and the total inelastic cross sections. The ${ }^{48} \mathrm{Ti}$ experimental results were compared with previous reported results, theoretical calculations performed using the TALYS 1.8 code and evaluated nuclear data libraries. 


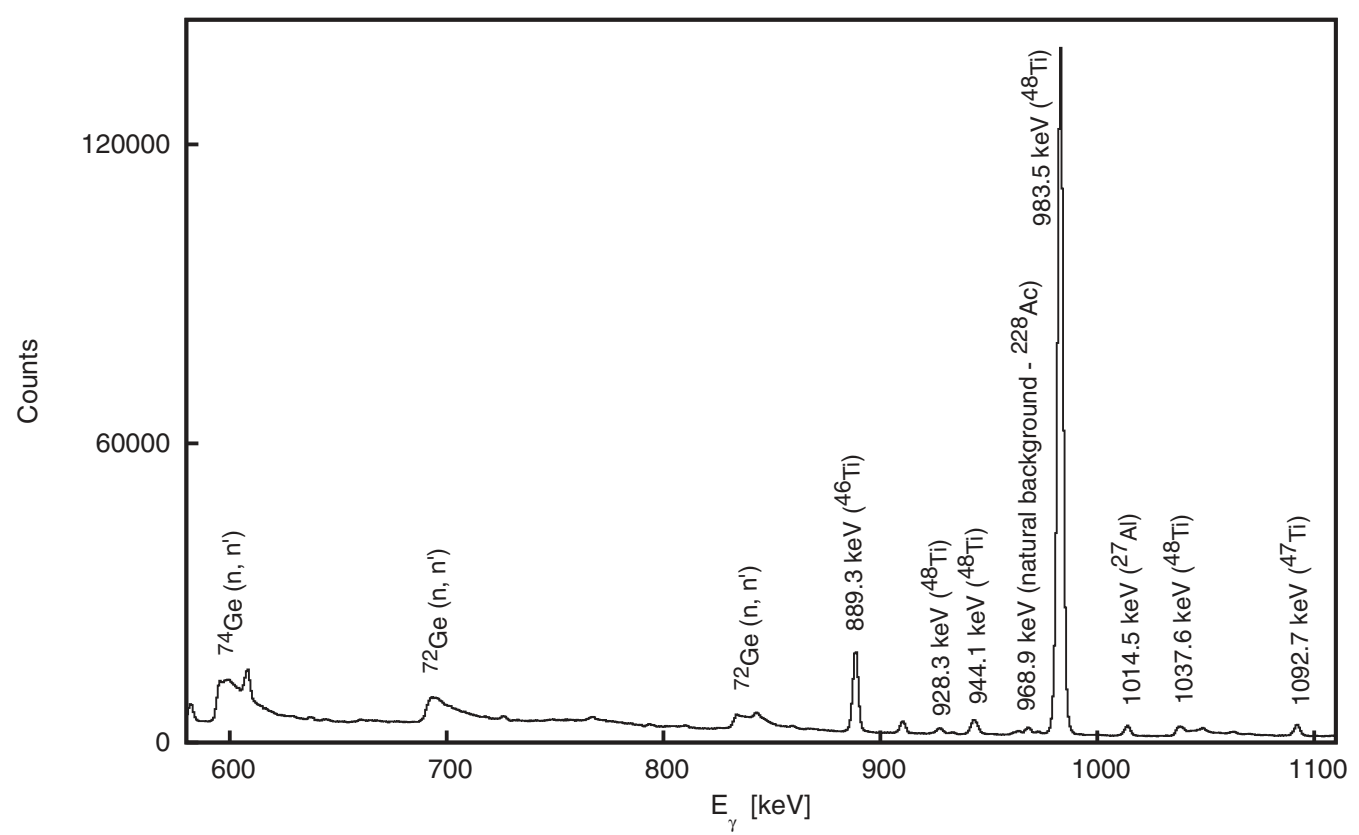

FIG. 9. The amplitude spectrum of one HPGe detector used in the present experiment with a zoom to highlight the 983.5-keV $\gamma$ ray and the peaks around it.

Overall, we obtained a reasonably good agreement with previous reported results in all the incident energy range except for a few points between $8-10 \mathrm{MeV}$. For the minor isotopes $\left({ }^{46,47,49,50} \mathrm{Ti}\right)$ we are the first to report experimental data. The reported $\gamma$ production cross sections have very low uncertainties (around or below 5\%) for the main transition in each isotope, and around $10 \%$ for the weaker ones for all the incident neutron energy range.

As a byproduct we investigated the decay of two levels in ${ }^{48} \mathrm{Ti}$ and found no support for the assignment included in TALYS. Eliminating the levels from the calculations improves the agreement with the data.

Finally, with regard to the possibility of using the first $\gamma$ ray from ${ }^{48} \mathrm{Ti}$ as a cross section standard, we note the remarkable agreement between our results and the data previously reported by Dashdorj et al. for a large energy range. This level of agreement between two independent measurements together with other favorable arguments (a background free $\gamma$ spectrum around the $\gamma$ energy of interest $-983.5 \mathrm{keV}$, the flat shape of the cross section, the simplicity to produce the sample) favor the first transition in ${ }^{48} \mathrm{Ti}$ as a choice for a standard cross section.

\section{ACKNOWLEDGMENTS}

This work was supported by the European Commission within the Seventh Framework Programme through Fission2013-CHANDA (Project No. 605203). The authors would like to thank the GELINA operators for their help and support during the measurements. M.B. would also like to acknowledge the POSDRU Grant No. POSDRU/159/1.5/S/137750 for $\mathrm{PhD}$ and postdoctoral students, while A.O. acknowledges the support received from the Romanian Ministry of Education through Contract No. PNII-RU-TE-2014-4-2003.
[1] BP Statistical Review of World Energy, 64th ed., June (2015).

[2] The Generation IV International Forum, online: http://www.gen4.org/.

[3] M. Salvatores et al., in Proceedings of the International Conference on Nuclear Data for Science and Technology, edited by O. Bersillon, F. Gunsing, E. Bauge, R. Jacqmin, and S. Leray, Nice, France (EDP Sciences, 2007), p. 878.

[4] Nuclear Energy Agency, OECD, Nuclear Data High Priority Request List, online: http://www.nea.fr/dbdata/hprl/.

[5] A. D. Carlson, V. G. Pronyaev, F.-J. Hambsch, W. Mannhart, A. Mengoni, R. O. Nelson, P. Talou, S. Tagesen, and H. Vonach, J. Korean Phys. Soc. 59, 1390 (2011).

[6] S. P. Simakov, V. G. Pronyaev, R. Capote, and R. O. Nelson, in Proceeding of the 13th International Conference on Nuclear
Reaction Mechanisms, edited by F. Cerutti, M. Chadwick, A. Ferrari, T. Kawano, S. Bottoni, and L. Pellegri (Villa Monastero, Varenna, Italy, 2012) CERN-Proceedings-2012-002, p. 321.

[7] A. D. Carlson et al., Nucl. Data Sheets 118, 126 (2014).

[8] A. D. Carlson et al., Nucl. Data Sheets 123, 27 (2015).

[9] A. D. Carlson et al., Eur. Phys. J. Web Conf. 106, 04002 (2016).

[10] M. Nyman, F. Belloni, D. Ichinkhorloo, E. Pirovano, A. J. M. Plompen, and C. Rouki, Phys. Rev. C 93, 024610 (2016).

[11] D. L. Broder, A. G. Dovebenko, V. E. Kolesov, A. I. Lashuk, and I. P. Sadokhin, preprint FEI-32, Obninsk, 19665, EXFOR 40035.

[12] M. V. Pasechnik, M. B. Fedorov, T. I. Jakovenko, I. E. Kashuba, and V. A. Korzh, Ukr. Fiz. Zh. 14, 1874 (1969). 
[13] W. Breunlich and G. Stengl, Nucl. Phys. A 184, 253 (1972).

[14] E. S. Konobeevskij, R. M. Musaelyan, V. I. Popov, V. M. Prokopenko, and I. V. Surkova, Izv. Ross. Akad. Nauk, Ser. Fiz. 37, 1764 (1973).

[15] W. E. Kinney and F. G. Perey, Oak Ridge National Lab. Report No. 4810 (1973).

[16] E. Barnard, J. Devilliers, D. Reitmann, P. Moldauer, A. B. Smith, and J. Whalen, Nucl. Phys. A 229, 189 (1974).

[17] I. A. Korzh, V. A. Mishchenko, E. N. Mozhzhukhin, N. M. Pravdivij, and I. E. Sanzhur, Ukrainskij Fizicheskij Zhurnal 22 (1977).

[18] A. Smith, P. Guenther, P. Moldauer, and J. Whalen, Nucl. Phys. A 307, 224 (1978).

[19] A. I. Lashuk, A. I. Gonchar, and I. P. Sadokhin, Vop. At. Nauki i Tekhn., Ser. Yadernye Konstanty 1994, 26 (1994).

[20] D. Dashdorj et al., Nucl. Sci. Eng. 157, 65 (2007).

[21] D. Dashdorj et al., Phys. Rev. C 75, 054612 (2007).

[22] N. Otuka et al., Nucl. Data Sheets 120, 272 (2014).

[23] A. Bensussan and J. M. Salome, Nucl. Instrum. Methods 155, 11 (1978).

[24] L. C. Mihailescu, L. Olah, and A. J. M. Plompen, Nucl. Instrum. Methods Phys. Res. A 531, 375 (2004).

[25] D. Ene, C. Borcea, S. Kopecky, W. Mondelaers, A. Negret, and A. J. M. Plompen, Nucl. Instrum. Methods Phys. Res. A 618, 54 (2010).

[26] D. Deleanu, C. Borcea, Ph. Dessagne, M. Kerveno, A. Negret, A. J. M. Plompen, and J. C. Thiry, Nucl. Instrum. Methods Phys. Res. 624, 130 (2010).

[27] A. Negret, C. Borcea, Ph. Dessagne, M. Kerveno, N. Nankov, A. Olacel, A. J. M. Plompen, and C. Rouki, Nucl. Data Sheets 119, 179 (2014).

[28] C. Rouki et al., Nucl. Instrum. Methods Phys. Res. A 672, 82 (2012).
[29] A. Olacel et al., Proceedings of International Conference on Nuclear Data for Science and Technology, Bruges, Belgium, 2016, submitted to EPJ Web Conf.

[30] J. Meija et al., Pure Appl. Chem. 88, 293 (2016).

[31] A. J. Koning, S. Hilaire, and M. C. Duijvestijn, in Proceedings of International Conference on Nuclear Data for Science and Technology, edited by O. Bersillon, F. Gunsing, E. Bauge, R. Jacqmin, and S. Leray, Nice, France (EDP Sciences, 2007), p. 211.

[32] A. Koning and J. Delaroche, Nucl. Phys. A 713, 231 (2003).

[33] A. Gilbert and A. G. W. Cameron, Can. J. Phys. 43, 1446 (1965).

[34] A. V. Ignatyuk, G. N. Smirenkin, and A. S. Tishin, Sov. J. Nucl. Phys. 21, 255 (1975).

[35] J. Kopecky and M. Uhl, Phys. Rev. C 41, 1941 (1990).

[36] D. M. Brink, Ph.D. thesis, Oxford University, 1955.

[37] P. Axel, Phys. Rev. 126, 671 (1962).

[38] R. Capote et al., Nucl. Data Sheets 110, 3107 (2009).

[39] T. W. Burrows, Nucl. Data Sheets 107, 1747 (2006).

[40] A. Higashi, K. Katori, M. Fujiwara, H. Ikegami, I. Katayama, S. Morinobu, M. Tosaki, S. I. Hayakawa, N. Ikeda, and H. Miyatake, Phys. Rev. C 39, 1286 (1989).

[41] T. Guhr, K.-D. Hummel, G. Kilgus, D. Bohle, A. Richter, C. W. De Jager, H. De Vries, and P. K. A. De Witt Huberts, Nucl. Phys. A 501, 95 (1989).

[42] M. B. Chadwick et al., Nucl. Data Sheets 112, 2887 (2011).

[43] A. Santamarina et al., JEFF Report 22, OECD/NEA Data Bank (2009)

[44] S.-C. Wu, Nucl. Data Sheets 91, 1 (2000).

[45] T. W. Burrows, Nucl. Data Sheets 108, 923 (2007).

[46] T. W. Burrows, Nucl. Data Sheets 109, 1879 (2008).

[47] Z. Elekes and J. Timar, Nucl. Data Sheets 112, 1 (2011).

[48] Z. G. Ge, Z. X. Zhao, H. H. Xia, Y. X. Zhuang, T. J. Liu, J. S. Zhang, and H. C. Wu, J. Korean Phys. Soc. 59, 1052 (2011). 